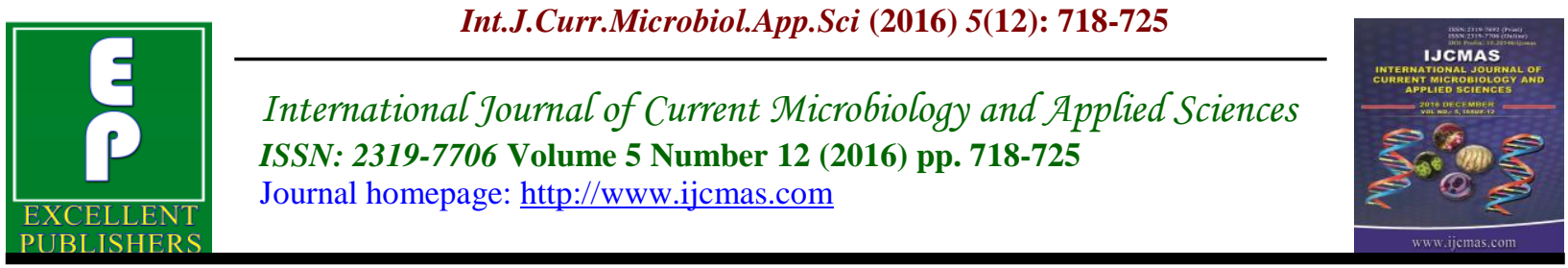

Original Research Article

http://dx.doi.org/10.20546/ijcmas.2016.512.082

\title{
Needle Stick Injuries among Health Care Worker at Tertiary Care Hospital
}

\author{
Kishor Ingole, Suwarna Pawar* and Sarika Pathak \\ Department of Microbiology, Dr V M Govt. Medical College, Solapur, Dist Solapur, \\ Maharashtra, 413003, India \\ *Corresponding author
}

\section{Keywords}

Needle stick injury, health care workers, post-exposure prophylaxis.

\section{Article Info}

Accepted:

26 November 2016

Available Online:

10 December 2016

\section{A B S T R A C T}

Health care workers (HCW) are at risk of an occupational exposure to blood borne most commonly by percutaneous injuries with sharp objects. This study was conducted to find out the occurrence of NSI's among HCW in a tertiary care hospital. A retrospective study was conducted in Department of microbiology at a tertiary care hospital in which data of all self-reported documents related to NSI for the period from 2013-2015 was collected and analyzed to determine the group of the HCW suffering the injury with details of the incident including mode of injury, different majors followed after injury and the status of post-exposure prophylaxis. Out of total $1295 \mathrm{HCW}, 15(1.16 \%)$ have reported the incidence of NSI's in 2013, $14(1.08 \%)$ in $2014 \& 17(1.31 \%)$ in 2015 . Among these total $46 \mathrm{HCW}$, interns were the most commonly injured group followed by doctors $(7.08 \%)$, laboratory technicians $(3.51 \%)$, nurses $(0.9 \%) \&$ servants $(0.3 \%)$. Recapping of used needle was most common mode of injury 19 (41.31\%) followed by suturing, splashing over open wound or mucous membrane or eyes, while IV catheterization or drawing out of blood, during drug administration \& handling of biomedical waste. Most of HCW's had washed the site with spirit or betadine. Among 46 HCW's 27 (58.70\%) had completed the PEP. Understanding the epidemiology of NSI's is very important to implement control measures. Compulsory clinical training of medical students in their academic is a must to prevent NSI's.

\section{Introduction}

Health care workers $(\mathrm{HCW})$ are at increased risk of infection with blood-borne pathogens because of occupational exposure (Bhattacharya et al., 2014). Any healthcare worker handling sharp devices is at risk of an occupational exposure to blood borne pathogens (Lukianskyte et al., 2011). Bloodborn viruses (BBVs) in particular hepatitis B (HBV), hepatitis C (HCV), and HIV can be transmitted occupationally from infected staff to patients, from infected patients to staff, or from patient to patient via contaminated (Dental et al., 2011). The risk of transmission of from patient to the healthcare worker is as follows: Hepatitis $\mathrm{C}$ (3\%), Hepatitis B (30\%), and HIV (0.3\%), which depends on the viral load of the patient (Suparna, 2016). Most exposures among HCWs are caused by percutaneous injuries with sharp objects contaminated with blood or body fluids which include needles, scalpels, lancets and broken glass. 
Needle stick injuries (NSIs) are wounds caused by sharps such as hypodermic needles, blood collection needles, intravenous (IV) cannulae or needles used to connect parts of IV delivery systems (Mandya et al., 2014). The causes include various factors like type and design of needle, recapping activity, handling/transferring specimens, collision between HCWs or sharps, during clean-up, manipulating needles in patient line related work, passing/handling devices or failure to dispose of the needle in puncture proof containers (Radha et al., 2012).

According to the Centers for Disease Control and Prevention, approximately 384,000 percutaneous injuries occur annually in US hospitals. EPInet data for 2003 reports a rate of approximately 27 NSIs per 100 beds in teaching hospitals. It is also found that $37.6 \%$ of Hepatitis B, 39\% of Hepatitis $\mathrm{C}$ and $4.4 \%$ of HIV/AIDS in Health-Care Workers around the world are due to needle stick injuries (Jaybhaye $e t$ al., 2014). More than $90 \%$ of these infections occur in developing countries. Needle stick injuries present the single greatest risk to medical personnel. Most of the NSI's remains unreported in developing countries.

This study is conducted to find out the occurrence of NSI's among health care worker in this tertiary care hospital.

\section{Materials and Methods}

The objective of this study was to determine the occurrence of needle stick injuries in health care workers at tertiary care hospital. Needle stick injuries are recorded in the casualty department. A retrospective study of all self-reported documents related to needle stick injuries for the period of two years from 2013 to2015 was conducted in Department of microbiology at a tertiary care hospital. The data was analyzed to determine the group of the health care worker suffering the injury \& the details of the incident, which included mode of injury, different majors which had followed after injury and the status of post-exposure prophylaxis.

\section{Results and Discussion}

Out of total 1295 health care workers, 15 $(1.16 \%)$ have reported the incidence of NSI's in 2013, 14 (1.08\%) have reported in 2014, 17 (1.31\%) have reported in 2015.

Among these 46 health care workers who were injured by needle sticks, interns were the most commonly injured group of workers which constituted $22.45 \%$ (22/98) of all the cases reported. About $7.08 \%$ $(16 / 226)$ of the incidents were reported in doctors, $3.51 \% \quad(2 / 57)$ were among laboratory technicians, $0.9 \% \quad(5 / 580)$ incidents were in nurses while $0.3 \%(1 / 334)$ was reported in servants. (Table 1, Figure 1)

When encountered about mode of injury, 19 $(41.31 \%)$ injuries were occurred while recapping, $11(23.91 \%)$ were occurred while surgical procedures like suturing. Splashing over open wound or mucous membrane or eyes was accounted for 7 (15.22\%) of cases while $6(13.04 \%)$ injuries were occurred while IV catheterization or drawing out of blood. Two (4.35\%) HCW's were injured during drug administration while only one $(2.17 \%)$ person was injured while handling of biomedical waste (Table 2, Figure 2).

After injury different majors have been followed, 22 (47.83\%) had washed with spirit or betadine, 14 (30.43\%) had washed the site with water and soap followed by spirit. Only water was used by $7(15.22 \%)$ people to wash the site while $3(6.52 \%)$ were ignored the injury (Table 3, Figure 3). 
Out of total 46 HCW's $27(58.70 \%)$ had completed the PEP, while the remaining 19 $(41.30 \%)$ were failed to complete it. Among the total 16 doctors, 11 completed the postexposure prophylaxis while 5 didn't complete the PEP. Out of total 22 interns 12 had completed the PEP while 10 were failed to complete the PEP. Only 3 out of 5 nurses completed the PEP while 2 failed to receive the PEP. Among 2 lab technicians one had completed while one didn't complete the PEP. Only one servant got NSI who failed to complete PEP (Table 4, Figure 4).

The present study highlights the situation of NSI in tertiary care hospital. Most of the health care workers had experienced the NSI at least once on their life.

In present study interns were the most commonly injured group of workers which constituted $22.45 \%(22 / 98)$ of all the cases reported. About $7.08 \%(16 / 226)$ of the incidents were reported in doctors, $3.51 \%$ (2/57) were among laboratory technicians, $0.9 \%(5 / 580)$ incidents were in nurses while $0.3 \%(1 / 334)$ was reported in servants. In a study done by Verma et al., also reported the high incidents among interns followed by doctors. Laishram et al., (2013), Jayanth et al., (2009), Verma et al., Jaybhaye et al., (2014), Jahnavi et al., (2014), Bhattacharya et al., (2014), Salelkar et al., (2010) were reported higher NSI's among nurses, while in present study interns were the most commonly injured group. The possible reason for this may be underreporting and lesser knowledge among the nurses as compared to interns and doctors. Overload and long working hours might be the reason for higher incidents among interns and doctors.

When encountered about mode of injury among health care workers in present study, there are various activities which lead to
NSI's these included recapping of needle after use, surgical procedures like suturing, splashing over open wound or mucous membrane or eyes \& also injuries while IV catheterization, drawing of blood, drug administration etc. The most common cause of injury was recapping of needle after use which was observed in $17(41.30 \%)$ HCW's. In studies done by Verma et al., Jahnavi et al., Jaybhaye et al., Bhattacharya et al., Lukianskyte et al., Radha et al., found recapping as the most common cause of needle stick injury. Second most common mode of injury was suturing which accounts for $11(23.91 \%)$ health care workers. Similar finding was also noted in Jayanth et al., (2009) \& Jayanth et al., (2013) in which suturing was constituted in $21.3 \%$ and $22 \%$ of HCW's respectively. In a study performed by Salekar et al., (2010) 46\% of injuries were occurred because of suturing. IV catheterization or drawing out of blood was the next reason for injuries which was seen in $6(13.04 \%)$ HCW's in present study. In a study done by Salekar also found the similar findings in which $13.8 \%$ of injuries were occurred while collecting blood sample. Laishram et al., (2013) also observed that $15.1 \%$ of injuries were occurred because of blood withdrawal. There were some other mode of injuries including splashing over open wound or mucous membrane or eyes which was accounted in 7 (15.21\%) of HCW's. Among $2(4.34 \%)$ personnel injury was occurred during drug administration while one $(2.17 \%) \mathrm{HCW}$ was injured while handling of biomedical waste.

In this study there are various majors which have been followed by HCW's after injury. Spirit and betadine was applied over the site by $22(47.83 \%)$ HCW's, 14 (30.43\%) had washed the site with water and soap followed by application of spirit. Only water was used by $7(15.22 \%)$ people to wash the 
site while $3(6.52 \%)$ were ignored the injury without taking any major. Similar observation was found in a study conducted by Salekar et al., where $71.5 \%$ of HCW's applied antiseptic lotion while 52\% HCW's immediately washed the site with soap and water and. Another study performed by Kaur et al., (2014) in nursing personnel had observed that maximum $72.8 \%$ washed the injured part with antiseptic solution and remaining $12.6 \%$ washed only with soap and water. There is one more study which was also carried out among the nursing personnel in which $70 \%$ of the personnel disinfected the wound, $5.6 \%$ washed the site with soap and water while the remaining $5.7 \%$ had squeezed the wound place. Verma et al., found that most of HCW's $53.5 \%$ had washed the site with soap and water, $41 \%$ had applied betadine and spirit at the site and $1 \%$ had done nothing about the injury the remaining had done the things like applying pressure, expressing the blood from the site, tying the part.

Table.1 Occurrence of needle stick injuries in health care workers.

\begin{tabular}{|c|c|c|c|}
\hline Sr No & Group of HCW & Number /Out of Total & Percentage \\
\hline 1 & Interns & $22 / 98$ & $22.45 \%$ \\
\hline 2 & Doctors & $(16 / 226)$ & $7.08 \%$ \\
\hline 3 & Lab Technicians & $(2 / 57)$ & $3.51 \%$ \\
\hline 4 & Nurses & $(5 / 580)$ & $0.9 \%$ \\
\hline 5 & Servants & $(1 / 334)$ & $0.3 \%$ \\
\hline \multicolumn{2}{|c|}{ Total } & $46 / 1295$ & $3.55 \%$ \\
\hline
\end{tabular}

Table.2 Circumstances of occurrence of NSI's.

\begin{tabular}{|c|c|c|c|}
\hline Sr No & Mode of Injury & Number & Percentage \\
\hline 1 & Recapping of Needle & 19 & 41.31 \\
\hline 2 & Suturing & 11 & 23.91 \\
\hline 3 & Splashing over open wound or mucous membrane or eyes & 7 & 15.22 \\
\hline 4 & IV catheterization or drawing of blood & 6 & 13.04 \\
\hline 5 & Drug administration & 2 & 4.35 \\
\hline 6 & Handling of Biomedical Waste & 1 & 2.17 \\
\hline & Total & 46 & 100 \\
\hline
\end{tabular}

Table.3 Different measures followed after NSI's:

\begin{tabular}{|c|c|c|c|}
\hline Sr No & Measures followed after injury & Number & Percentage \\
\hline 1 & Washed with spirit or betadine & 22 & 47.83 \\
\hline 2 & Washed the site with water and soap followed by spirit & 14 & 30.43 \\
\hline 3 & Washed with only water & 7 & 15.22 \\
\hline 4 & Ignored the injury & 3 & 6.52 \\
\hline & Total & 46 & 100 \\
\hline
\end{tabular}


Table.4 Status of Post exposure prophylaxis in HCW's:

\begin{tabular}{|c|c|c|c|}
\hline Sr No & Status of Post exposure prophylaxis in HCW's & Number & Percentage \\
\hline 1 & Completed PEP & 27 & 58.70 \\
\hline 2 & Failed to complete PEP & 19 & 41.30 \\
\hline & Total & 46 & 100 \\
\hline
\end{tabular}

Fig.1 Occurrence of needle stick injuries in health care workers.

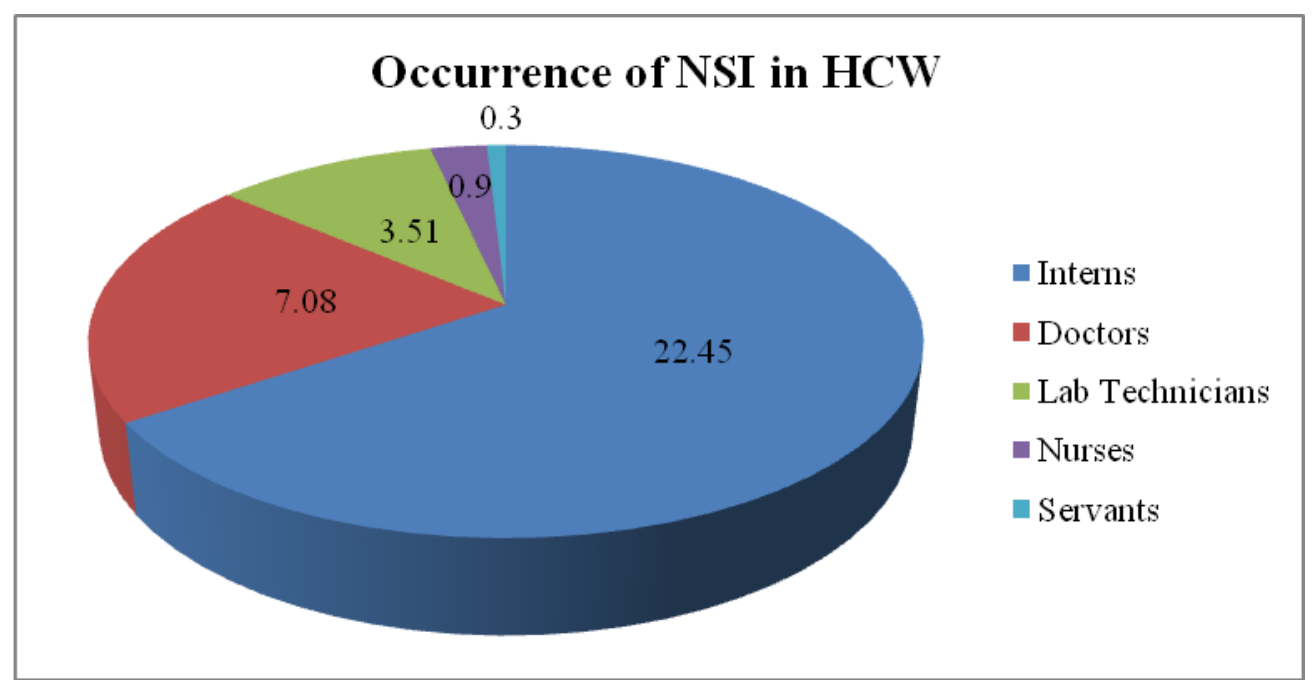

Fig.2 Circumstances of occurrence of NSI's.

\section{Circumstances of occurrence of NSI's among HCW's.}

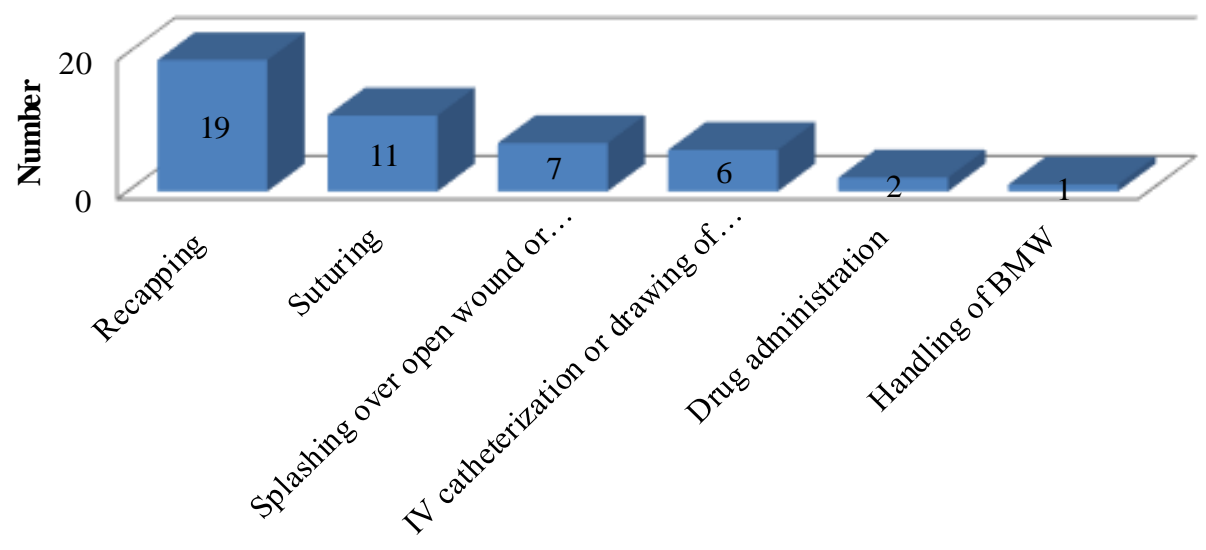

Mode of Injuries 
Fig.3 Different measures followed after NSI's.

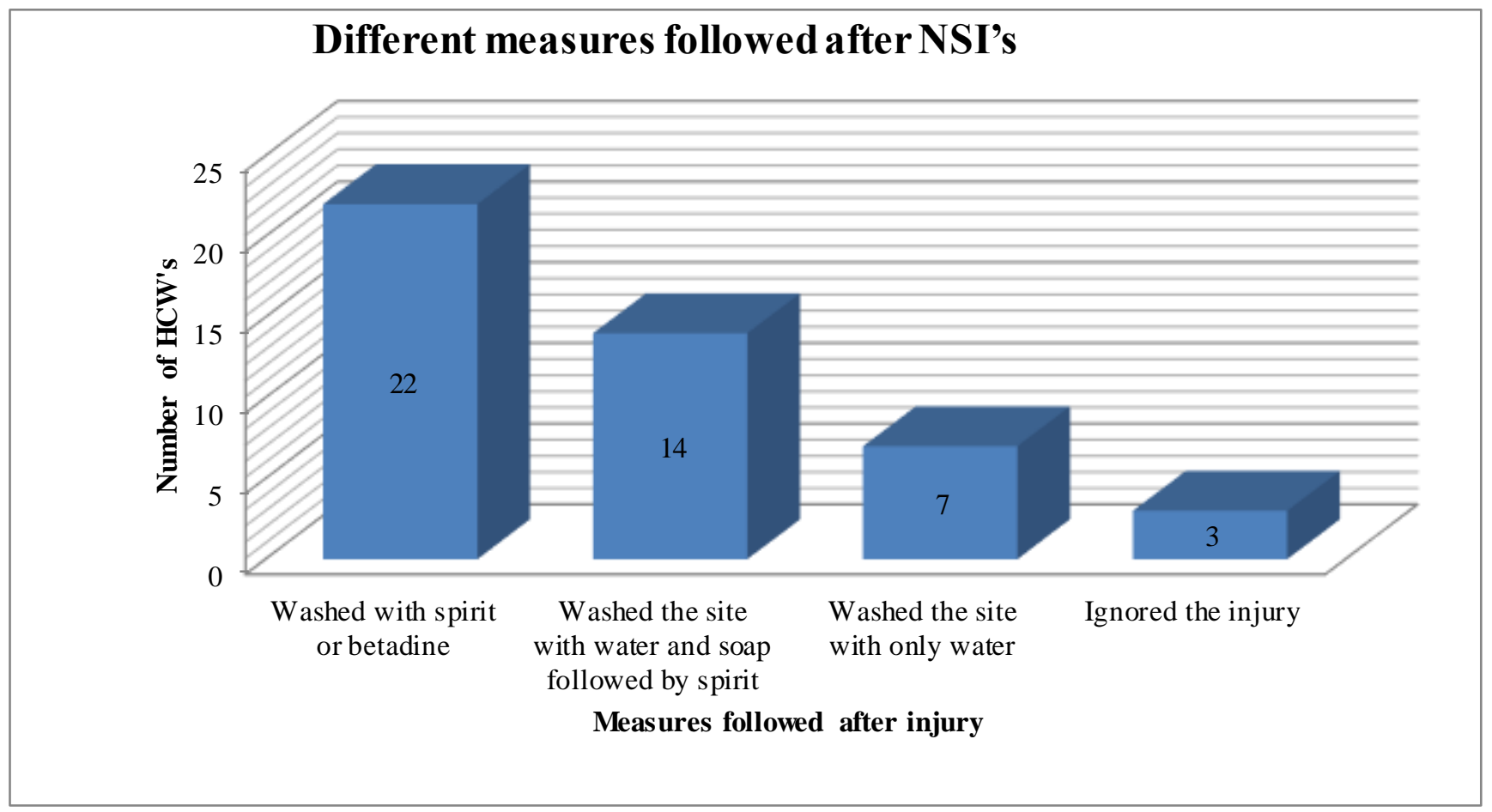

Fig.4 Status of Post exposure prophylaxis in HCW's.

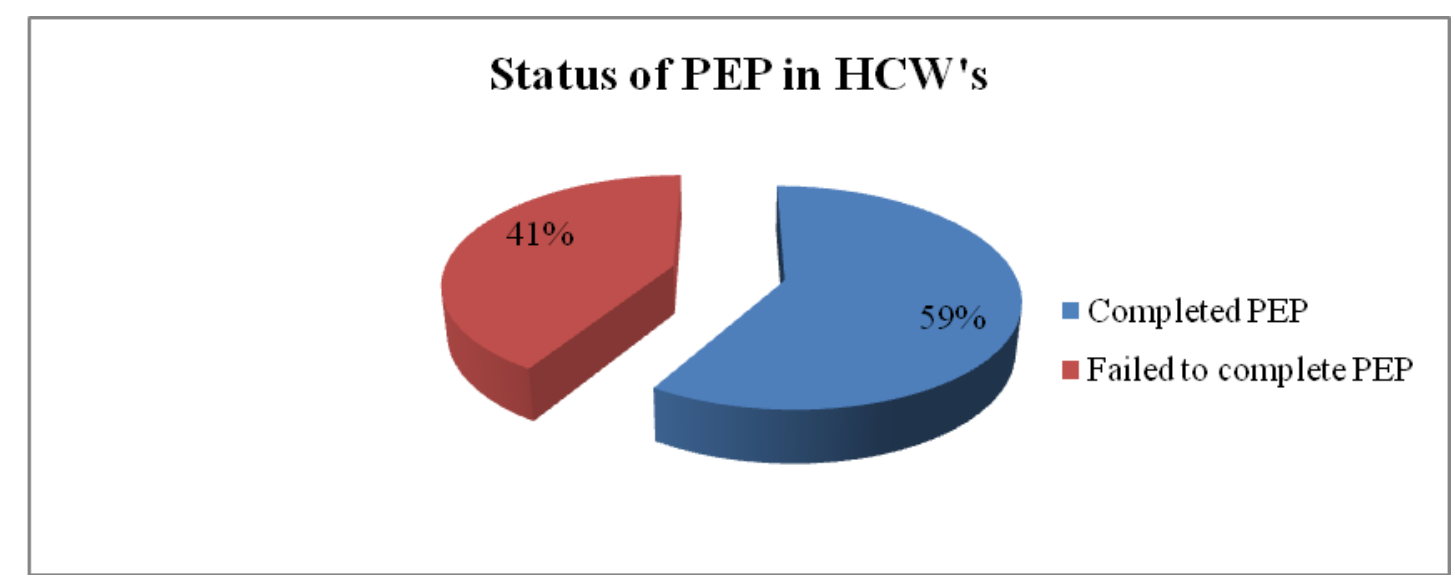

In present study out of total $46 \mathrm{HCW}$ 's 27 $(58.70 \%)$ had completed the post-exposure prophylaxis which included 11 doctors, 12 interns, 3 nurses and one lab technician. Nineteen $(41.30 \%)$ health care workers failed to complete post exposure prophylaxis in which there were 5 doctors, 10 interns, 2 nurses, 1 lab technician and one servant. Most of the interns and doctors have completed the post exposure prophylaxis. In a study by Shiva et al., (2011) at Tehran found that $32.57 \%$ had not completed their PEP while $67.43 \%$ had completed the PEP. Singru et al., (2008) found that $21.6 \%$ HCWs took PEP. It was found that only $3 \%$ HCW's had taken PEP in a study performed by Salekar et al. Post exposure prophylaxis is the most important step which should be 
followed after NSI. But different studies showed that most of the HCW's are still not completing the PEP. Lesser knowledge and awareness regarding needle stick injuries may be the reason for this.

In Conclusion, needle stick injuries are an important and continuing cause of exposure to serious and fatal diseases among health care workers. Understanding the epidemiology of NSI's is very important to implementing control measures. This study showed that most of needle stick injuries are found among interns. Compulsory clinical training of medical students in their academic is a must to prevent NSI's. Continuing education and training programs for hospital staff are required to keep them up to date.

\section{References}

Afridi Khan, A., Kumar, A., Sayani, R. 2013. Needle Stick Injuries - Risk and Preventive Factors: A Study among Health Care Workers in Tertiary Care Hospitals in Pakistan. Global J. Health Sci., 5(4): 86-92.

Bhattacharya, A., Basu, M., Das, P. 2014. The pattern of needle stick injury among health care workers at West Bengal. Muller J. Med. Sci. Res., 5(1): 29-33.

Dental, U.G. 2011. Jaber A Mohamed A. A survey of needle sticks and other sharp injuries among dental undergraduate students. Int. J. Infect. Control, 7(3): 1-10.

Jayanth, Jayanth, S.T., Kirupakaran, H., Brahmadathan, K.N., Gnanaraj, L., Kang, G. 2009. Needle stick injuries in a tertiary care hospital. Indian J. Med. Microbiol., 27(1): 44-7.

Jaybhaye. 2014. Rural India: Jaybhaye D., Dahire P., Nagaonkar A., Vedpathak V., Deo D., Kawalkar U. Needle stick injuries among health care workers in tertiary care hospital of rural India. Int. J. Med. Sci. Public Health, 3(1): 4952.

Kaur, Galougahi, M.H. 2010. Evaluation of needlestick injuries among nurses of Khanevadeh Hospital in Tehran. Iran J. Nurs. Midwifery Res., 15: 172-7.

Kaur, Kaur, D., Jaspal, S., Bajwa, S. 2014. Behavior, perception and compliance related to adoption of safety measures in response to needle stick injuries among nursing personnel at a tertiary care institute of North India. $J$. Scientific Society, 41( 1):32-37.

Laishram, Laishram, J., Keisam, A., Phesao, E., Shyami Tarao, M., Valerie J. L., H. Sanayaima Devi. 2013. Prevalence of needle stick injuries among nurses in a tertiary care hospital and their immediate response. Int. J. Med. Public Health, 3(4): 257-60.

Lukianskyte, R., Gataeva, J., Radziunaite, L. 2011. Nurses: Needle sticks and sharps injuries experienced by staff nurses and nursing students and their prevention. Int. J. Infect. Control, 8(1): 3-9.

Mandya Janhavi, Rajagopal, J., Manjunath, M., Mahendra, B.J., Ragini, R., Swetha, H.J., Harshitha, M.C., Nishitha, P. 2014. Needle Stick Injury among Health Care Workers in a Government Teaching Hospital, Mandya. Int. J. Scientific Study, 2(7):103-06.

Radha, R., Radha, R., Asif Khan. 2012. Epidemiology of needle sticks injuries among the health care workers of a rural tertiary care hospital -a crosssectional study. Nat. J. Community Med., 3(4): 589-94.

Salekar, Salelkar, S., Motghare, D., Kulkarni M, Vaz F. 2010. Study of Needle Stick Injuries among Health Care Workers at a Tertiary Care 
Hospital. Indian J. Public Health, 54(1), 18-20.

Shiva, Farideh, S., Sanaei, A., Shamshiri, A., Ghotbi, F. 2011. Survey of needlestick injuries in paediatric health personnel of 5 University Hospitals in Tehran. J. Pak. Med. Assoc., 61(2): 127-31.

Sigru, Singru, S.A., Banerjee, A. 2008. Occupational exposure to body fluids among health care workers in a teaching hospital in Mumbai, India. Indian J. Community Med., 33: 26-30.

Suparna, Perry, J., Parker, G., Jagger, J. 2005. EPINET report: 2003 percutaneous injury rates. $A d v$. Exposure Prev., 7: 2-45.

Suparna. 2016. The National Survelliance System for Healthcare Workers (NaSH) Summary Report for Blood and Body Fluid Exposure (19952007). Retrieved August, 2016 from http://www.cdc.gov/nhsn/PDFs/NaSH/ NaSH-Report-6-2011.pdf

Verma, Verma, y., Bandlish, D., Kumar, B. The prevalence of needle stick injury among healthcare personnel in a tertiary care hospital in Kolkata. http://www.webmedcentral.com. 1-8

\section{How to cite this article:}

Kishor Ingole, Suwarna Pawar and Sarika Pathak. 2016. Needle Stick Injuries among Health Care Worker at Tertiary Care Hospital. Int.J.Curr.Microbiol.App.Sci. 5(12): 718-725. doi: http://dx.doi.org/10.20546/ijcmas.2016.512.082 